\title{
EDITORIAL
}

\section{WITH A LITTLE HELP FROM YOUR FRIENDS...}

You can develop drugs alone, but given the complexity of the systems under study, it is easier to combine expertise. Intellectual property concerns mean that industry's contribution to the chorus

of voices advocating integration can sometimes sound a little off-key.

"Where observation is concerned, chance favours only the prepared mind", as famously said by Louis Pasteur at the inauguration of Lille University's Faculty of Science almost 150 years ago. The phrase conjures up visions of absent-minded nineteenth-century polymaths experiencing moments of unexpected revelation, such as when Paul Ehrlich accidentally left a preparation of bacilli on the stove, which resulted in the heat-induced disintegration of their fatty coating and led to the discovery of his universally applied staining method. In these days of increasing scientific specialization, the observation is perhaps just as likely to come from an interested colleague from a related discipline popping their head round the office door and making an insightful remark, which hopefully your mind is prepared enough not to ignore.

However, the unpreparedness of minds too focused on their particular speciality is an increasingly worrying phenomenon in biological research. Attempts are underway to train a new generation of interdisciplinary scientists with the skills to get their heads around the multiple techniques that need to be applied to crack the toughest of biological nuts. Cross-disciplinary training lies at the heart of initiatives such as Oxford University's integrative ion channel research programme (described in this month's Career's Focus on page 679) and the new Broad Institute in Cambridge, Massachusetts, which will bring together the bioscience strengths of Harvard, the Massachusetts Institute of Technology and the Whitehead Institute. It is academic ventures such as these that are at the forefront of efforts to re-integrate an increasingly fragmented biology.

Mindful of the benefits of interdisciplinary integration, most drug discovery companies try to factor in such interactions whenever they build a new research facility. They place the chemists alongside the screeners, for instance, so that they are more likely to bump into each other when they go for coffee, giving chance more opportunities to work its magic. In a sense, Nature Reviews Drug Discovery tries to reproduce that architecture in print. By mixing up the subjects in an accessible way, we hope that while browsing through for the articles relevant to your own area you might just happen upon that piece of information from another field that sets you to thinking.

Another sort of integration we attempt in the journal is the mixing of academic and industrial perspectives, not least because the lengthening list of failed drug development programmes is highlighting the fact that the biology underlying drug discovery is far more complex than people seem to have imagined. We're learning that in many areas we simply don't yet know enough to jump straight to the drug. With fundamental research less and less a part of the industrial research landscape, and decisions about which projects to pursue increasingly being made by nonscientists, more dialogue between academic and industrial researchers is needed to bring this scientific complexity (or reality) to the fore.

Desirable as such dialogue is perceived to be, industry's preoccupation with secrecy is a major barrier. A scene from a high-profile conference last month illustrates the difficulty. An industry speaker presenting a new in-house in situ database invited academic collaborators to submit data in return for access to the collected information - an appealing prospect, as much of the data it contains is unpublished, but with the next breath the speaker refused to reveal the structure of the compound used to generate the images he was using to illustrate the database. Hardly a great selling job.

Given the mounting evidence indicating that the industry's mantra about the therapeutic prospects for many diseases is out of tune with the academic view of those slices of biology, it makes sense for industry to make as much use of its potential friends outside the walls as possible. That means opening up the channels of communication and seeing the benefits of freely sharing knowledge for the sake of it, without clear goals. Pasteur also said, "There are no such things as applied sciences, only applications of science", suggesting that we're all in this together. 